TRANSACTIONS OF THE

AMERICAN MATHEMATICAL SOCIETY

Volume 360, Number 8, August 2008, Pages 4263-4285

S 0002-9947(08)04402-4

Article electronically published on March 20, 2008

\title{
TOWARDS A UNIVERSAL SELF-NORMALIZED MODERATE DEVIATION
}

\author{
BING-YI JING, QI-MAN SHAO, AND WANG ZHOU
}

\begin{abstract}
This paper is an attempt to establish a universal moderate deviation for self-normalized sums of independent and identically distributed random variables without any moment condition. The exponent term in the moderate deviation is specified when the distribution is in the centered Feller class. An application to the law of the iterated logarithm is given.
\end{abstract}

\section{INTRODUCTION AND MAIN RESULTS}

Let $X, X_{1}, X_{2}, \ldots$ be independent and identically distributed (i.i.d.) nondegenerate random variables. Put

$$
S_{n}=\sum_{i=1}^{n} X_{i}, \quad V_{n}^{2}=\sum_{i=1}^{n} X_{i}^{2}
$$

and define the self-normalized sum by $S_{n} / V_{n}$. In contrast to the classical limit theorems, the self-normalized limit theorems enjoy much better properties with few or no moment conditions. Griffin and Kuelbs [10] obtained a self-normalized law of the iterated logarithm for all distributions in the domain of attraction of a normal or stable law. Shao 14 showed that no moment conditions are needed for a self-normalized large deviation result: If $E X=0$ or $E X^{2}=\infty$, then for $0<x<1$,

$$
\lim _{n \rightarrow \infty} P\left(S_{n} / V_{n} \geq x \sqrt{n}\right)^{1 / n}=\exp \left\{-\lambda\left(x^{2}\right)\right\}
$$

where $\lambda(x)=\inf _{b \geq 0} \sup _{t>0}\left(t x-\ln E \exp \left\{t\left(2 b X-b^{2} X^{2}\right)\right\}\right)$. It was also shown in Shao [14 that the tail probability of $S_{n} / V_{n}$ is Gaussian like when $X$ is in the domain of attraction of the normal law and sub-Gaussian like when $X$ is in the domain of attraction of a stable law. In particular, when $X$ is symmetric and in the domain of attraction of a stable law of order $\alpha(0<\alpha<2)$,

$$
\ln P\left(S_{n} / V_{n} \geq x_{n}\right) \sim-x_{n}^{2} \beta_{\alpha}
$$

Received by the editors February 7, 2006 and, in revised form, August 14, 2006.

2000 Mathematics Subject Classification. Primary 60F10, 60F15; Secondary 60G50.

Key words and phrases. Moderate deviation, large deviation, self-normalized sums, the law of the iterated logarithm.

The first author was supported in part by Hong Kong RGC CERG No. HKUST6117/02P and DAG05/06.SC01.

The second author was partially supported by the National Science Foundation under Grant No. DMS-0103487 and HKUST DAG 05/06 Sc27 and RGC CERG No. 602206.

The third author was partially supported by the grants R-155-000-035-112 and R-155-050-055133/101 at the National University of Singapore. 
for any $x_{n} \rightarrow \infty$ satisfying $x_{n}=o(\sqrt{n})$, where $\beta_{\alpha}$ is the solution of

$$
\int_{0}^{\infty} \frac{2-\exp \left\{2 x-x^{2} / \beta\right\}-\exp \left\{-2 x-x^{2} / \beta\right\}}{x^{\alpha+1}} d x=0 .
$$

Here and in the sequel, $a_{n} \sim b_{n}$ means $\lim _{n \rightarrow \infty} a_{n} / b_{n}=1$. Jing, Shao and Zhou 12 refined the self-normalized deviation and proved a self-normalized saddlepoint approximation without any moment assumption. Jing, Shao and Wang [11 established a Cramér type large deviation for self-normalized sums of independent random variables under a Lindeberg type condition. For other self-normalized results we refer to Logan, Mallows, Rice and Shepp 13 for the limiting distribution of $S_{n} / V_{n}$ when $X$ is in the domain of attraction of a stable law, which has been proved also necessary by Götze and Chistyakov [8], Bentkus and Götze [1] for Berry-Esseen inequalities, Giné, Götze and Mason [6] for the necessary and sufficient condition for the asymptotic normality, Csörgo", Szyszkowicz and Wang [2, 3] for the Darling-Erdős theorem and Donsker theorem, and Shao [15, 16] for surveys on recent developments in this area.

The main aim of this paper is to establish a universal self-normalized moderate deviation without any moment condition. Results in (1.1) and (1.2) suggest that a very likely result would be

$$
\ln P\left(S_{n} / V_{n} \geq x_{n}\right) \sim-n \lambda\left(x_{n}^{2} / n\right)
$$

for $x_{n} \rightarrow \infty$ with $x_{n}=o(\sqrt{n})$. We shall prove that this is the case when $X$ is in the centered Feller class.

Before we state our main theorem, let us introduce some notation. Let $C_{s}$ denote the support of $X$, that is,

$$
C_{s}=\{x: P(X \in(x-\epsilon, x+\epsilon))>0 \text {, for any } \epsilon>0\} .
$$

We denote the number of elements in $C_{s}$ by $\operatorname{Card}\left(C_{s}\right)$ and define $\operatorname{Card}\left(C_{s}\right)=\infty$ if $C_{s}$ does not contain a finite number of elements. The random variable $X$ is said to satisfy condition $(H 1)$ if

$$
C_{s} \cap R^{+} \neq \emptyset \text { and } C_{s} \cap R^{-} \neq \emptyset \text {, where } R^{+}=\{x: x>0\}, R^{-}=\{x: x<0\}
$$

and to satisfy condition $(H 2)$ if

$$
E X=0 \text { or } E X^{2}=\infty .
$$

We say $X$ is in the centered Feller class, introduced by Giné and Mason [7, if there exists a sequence of norming constants $\left\{a_{n}\right\}_{n \geq 1}$ such that for every subsequence of $\{n\}$ there exists a further subsequence $\left\{n^{\prime}\right\}$ such that $S_{n^{\prime}} / a_{n^{\prime}}$ converges in distribution to a nondegenerate law. Giné and Mason [7] also showed that $X$ is in the centered Feller class if and only if $X \in \mathcal{F}_{\theta}$ for some $0 \leq \theta<\infty$, where

$$
\mathcal{F}_{\theta}=\left\{X: \limsup _{a \rightarrow \infty} \frac{a^{2}\left\{P(|X|>a)+a^{-1}|E X I(|X| \leq a)|\right\}}{E X^{2} I(|X| \leq a)}=\theta\right\} .
$$

Theorem 1.1. Assume that $X$ satisfies conditions (H1) and (H2). Also assume that $X$ is in the centered Feller class. Then (1.4) holds for any sequence $\left\{x_{n}, n \geq 1\right\}$ with $x_{n} \rightarrow \infty$ and $x_{n}=o(\sqrt{n})$ as $n \rightarrow \infty$. If, in addition, $\operatorname{Card}\left(C_{s}\right) \geq 3$, then

$$
\lim _{n \rightarrow \infty} \frac{\ln P\left(\frac{S_{n}}{V_{n}} \geq x_{n}\right)}{x_{n}^{2}}=-t_{0}
$$


where $t_{0}=\lim _{x \rightarrow 0^{+}} t_{x}$, and $\left(t_{x}, b_{x}\right)$ satisfy the following saddlepoint equations:

$$
\begin{aligned}
E b\left(2 X-b X^{2}\right) \exp \left\{t b\left(2 X-b X^{2}\right)\right\} & =x E \exp \left\{t b\left(2 X-b X^{2}\right)\right\}, \\
E\left(X-b X^{2}\right) \exp \left\{t b\left(2 X-b X^{2}\right)\right\} & =0 .
\end{aligned}
$$

As will be proved in Proposition 2.2, $t_{0}$ is a positive and finite number. Theorem 1.1 together with the subsequence method is ready to give the following law of the iterated logarithm (LIL).

Theorem 1.2. Assume that $(H 1)$ and $(H 2)$ are satisfied and that $X$ is in the centered Feller class. Then

$$
\limsup _{n \rightarrow \infty} \frac{S_{n}}{V_{n} \sqrt{\log \log n}}=\frac{1}{\sqrt{t_{0}}} \text { a.s. }
$$

Remark 1.1. Theorem 1.2 is related to the main result in Giné and Mason [7. They show that the limsup in (1.9) is finite whenever

$$
\frac{S_{n}}{V_{n}}=O_{p}(1)
$$

Later, Griffin 9 proved that (1.10) holds if and only if

$$
\limsup _{a \rightarrow \infty} \frac{|a E X I(|X| \leq a)|}{a^{2} P(|X|>a)+E X^{2} I(|X| \leq a)}<\infty .
$$

Remark 1.2. Note that $E X^{2}$ is either infinite, which means that (H2) is satisfied, or finite, which implies that the mean must be finite. So assumption (H2) indeed doesn't require any moment condition. Assumption (H1) simply avoids the case where $X$ is nonnegative or nonpositive.

Remark 1.3. If $X$ is symmetric and in the domain of attraction of a stable law of order $\alpha(0<\alpha<2)$, then $t_{0}=\beta_{\alpha}$, where $\beta_{\alpha}$ is the solution of (1.3). In general, when $X$ is in the domain of attraction of a stable law, $t_{0}$ does equal the constant $\beta\left(\alpha, c_{1}, c_{2}\right)$ given in Theorem 3.2 of Shao [14.

This paper is organized as follows. In the next two sections we will focus on some basic properties of the solution $\left(t_{x}, b_{x}\right)$ of saddlepoint equations (1.7) and (1.8), which may be of independent interest. Proofs of the main results will be postponed to Section 4.

\section{The SOlution to The SADDLEPOINT EQUATIONS}

This section is devoted to the study of some basic properties of the solution $\left(t_{x}, b_{x}\right)$ to the saddlepoint equations. Let

$$
g(t, b ; x)=t x-\ln E \exp \left\{t\left(2 b X-b^{2} X^{2}\right)\right\} .
$$

Our first property is that $\inf _{b \geq 0} \sup _{t \in R} g(t, b ; x)$ is attained at some finite unique points $t_{x}>0$ and $b_{x}>0$ for $x$ in a right neighborhood of zero.

Proposition 2.1. Assume that $(H 1)$ and $(H 2)$ are satisfied and $\operatorname{Card}\left(C_{s}\right) \geq 3$. Then there exists an $\epsilon_{0}>0$, such that for all $x \in\left(0, \epsilon_{0}\right), \inf _{b>0} \sup _{t \in R} g(t, b ; x)$ is attained at some finite unique points $t_{x}>0$ and $b_{x}>0$ and $\left(t_{x}, b_{x}\right)$ satisfy the saddlepoint equations (1.7) and (1.8).

To prove Proposition 2.1, we need the following lemmas. 
Lemma 2.1. Let $x>0$ and assume $\operatorname{Card}\left(C_{s}\right) \geq 3$. Then under $(H 2), g(t, b ; x)$ is strictly increasing in $t$ for $t \in\left(-\infty, \epsilon_{1}\right)$ for some $\epsilon_{1}>0$.

Proof. The proof follows the same lines of that of Lemma A.2 in Jing, Shao and Zhou [12. See the Appendix for a detailed proof.

For $0<x<1$, define

$$
\begin{aligned}
a_{10} & :=a_{10}(b)=\frac{1}{b}(1-\sqrt{1-x}), a_{20}:=a_{20}(b)=\frac{1}{b}(1+\sqrt{1-x}), \\
a_{1} & :=a_{1}(b)=\min \left(a_{10}, a_{20}\right), \quad a_{2}:=a_{2}(b)=\max \left(a_{10}, a_{20}\right)
\end{aligned}
$$

and

$$
U=\left\{b:\left(a_{1}(b), a_{2}(b)\right) \cap C_{s} \neq \emptyset\right\} .
$$

Lemma 2.2. Assume $\operatorname{Card}\left(C_{s}\right) \geq 3$ and $(H 2)$ is satisfied. Let $x \in(0,1)$.

(i) If $b \in U$, then

$$
\sup _{t \in R} g(t, b ; x)=\sup _{t>0} g(t, b ; x),
$$

and the supremum is attained at some finite unique point $\tilde{t}:=\tilde{t}(b, x)>0$ which is a finite unique solution to the equation $\partial g(t, b ; x) / \partial t=0$.

(ii) If $b \notin U$, then

$$
\sup _{t \in R} g(t, b ; x)=-\ln \left(P\left(X=a_{1}\right)+P\left(X=a_{2}\right)\right)
$$

and the supremum is attained at $\infty$.

Proof. We first note that $U \neq \emptyset$ by the fact that $\bigcup_{b}\left\{b:\left(a_{1}(b), a_{2}(b)\right)\right\}=R$. An elementary argument also yields that $U$ is an open set. (If $(H 1)$ holds, then $U \cap R^{+}$ is also a nonvoid open set.) Let

$$
h(y)=b^{2} y^{2}-2 b y+x=b^{2}\left(y-a_{1}\right)\left(y-a_{2}\right) .
$$

Suppose $b \in U$. Since

$$
\left(a_{1}, a_{2}\right) \cap C_{s} \neq \emptyset
$$

there must exist $W:=\left[a_{3}, a_{4}\right] \subset\left(a_{1}, a_{2}\right)$ so that

(a) there exists $\delta>0$ such that $h(y)<-\delta$ for each $y \in W$;

(b) $P(X \in W)>0$.

Then, as $t \rightarrow \infty$,

$$
\begin{aligned}
g(t, a ; b) & =-\ln E e^{-t h(X)} \leq-\ln E e^{-t h(X)} I(X \in W) \\
& \leq-\ln \left\{e^{t \delta} P(X \in W)\right\} \rightarrow-\infty,
\end{aligned}
$$

which combined with Lemma 2.1 shows that $\sup _{t \in R} g(t, b ; x)$ is attained at some finite $\tilde{t}=\tilde{t}(b, x)>0$. Since $g(t, b ; x)$ is a differentiable function of $t$ when $t>0$, we get that $\partial g(\tilde{t}, b ; x) / \partial t=0$. Also note that

$$
\frac{\partial^{2} g(t, b ; x)}{\partial t^{2}}=-\left(\frac{E Z^{2} e^{t Z}}{E e^{t Z}}-\left(\frac{E Z e^{t Z}}{E e^{t Z}}\right)^{2}\right)<0,
$$

where $Z=2 b X-b^{2} X^{2}$. There is at most one solution to the equation $\partial g(t, b ; x) / \partial t$ $=0$. Therefore $\tilde{t}$ is also unique. 
Next, suppose $b \notin U$. Since $C_{s}$ is necessarily closed, $\left[a_{1}, a_{2}\right] \cap C_{s}$ contains at most two points $\left\{a_{1}, a_{2}\right\}$. Clearly, we have

$$
h(y)>0 \text { for each } y \in C_{s} \backslash\left\{a_{1}, a_{2}\right\} .
$$

Therefore,

$$
\begin{aligned}
g(t, b ; x) & =-\ln E e^{-\operatorname{th}(X)} \\
& =-\ln \left(E e^{-t h(X)} I\left(X \in C_{s} \backslash\left\{a_{1}, a_{2}\right\}\right)+P\left(X=a_{1}\right)+P\left(X=a_{2}\right)\right) \\
& \nearrow-\ln \left(P\left(X=a_{1}\right)+P\left(X=a_{2}\right)\right) \quad \text { as } t \rightarrow \infty .
\end{aligned}
$$

Lemma 2.3. Assume that $\operatorname{Card}\left(C_{s}\right) \geq 3, R^{+} \cap C_{s} \neq \emptyset$ and that (H2) is satisfied. Then for $\epsilon_{0}$ sufficiently small and $x \in\left(0, \epsilon_{0}\right)$,

$$
\inf _{b>0} \sup _{t \in R} g(t, b ; x)=\inf _{b \in U \cap R^{+}} \sup _{t \in R} g(t, b ; x) .
$$

Proof. We use the contradiction method. Suppose (2.5) doesn't hold for $\epsilon_{0}$ sufficiently small. Then there exists a sequence $\left\{x_{k}\right\}_{k=1}^{\infty}$ in $(0,1)$ such that $x_{k} \rightarrow 0$ as $k \rightarrow \infty$ and

$$
\inf _{b>0} \sup _{t \in R} g\left(t, b ; x_{k}\right)=\inf _{b \in R^{+} \backslash U} \sup _{t \in R} g\left(t, b ; x_{k}\right)
$$

for $k \geq 1$. Since $\operatorname{Card}\left(C_{s}\right) \geq 3$, there exist $c_{1}, c_{2}, c_{3}$ and $\delta>0$ such that

$$
p_{i}:=P\left(X \in\left[c_{i}-\delta, c_{i}+\delta\right]\right)>0
$$

and the three intervals $\left[c_{i}-\delta, c_{i}+\delta\right], i=1,2,3$ are disjoint. Lemma 2.2 implies

$$
\begin{aligned}
& \sup _{b \in R^{+} \backslash U} \inf _{t \in R} E \exp \left\{t\left(2 b X-b^{2} X^{2}-x_{k}\right)\right\} \\
= & \exp \left(-\inf _{b \in R^{+} \backslash U} \sup _{t \in R} g\left(t, b ; x_{k}\right)\right) \\
= & \sup _{b \in R^{+} \backslash U} P\left(X=\frac{1}{b}\left(1-\sqrt{1-x_{k}}\right)\right)+P\left(X=\frac{1}{b}\left(1+\sqrt{1-x_{k}}\right)\right) \\
\leq & 1-\min \left(p_{1}, p_{2}, p_{3}\right)<1 .
\end{aligned}
$$

To get a contradiction, it suffices to show that

$$
\sup _{b \in R^{+} \backslash U} \inf _{t>0} E \exp \left\{t\left(2 b X-b^{2} X^{2}-x_{k}\right)\right\} \geq 1
$$

Let $p=P(X>0)$. Since $R^{+} \cap C_{s} \neq \emptyset$, we have $p>0$. Put $b_{k}=\sqrt{x_{k}}$. Then

$$
P\left(6 x_{k} /\left(p b_{k}\right) \leq X \leq 1 / b_{k}\right) \rightarrow p \text { and } P\left(-x_{k} / b_{k} \leq X \leq 0\right) \rightarrow 1-p
$$


and

$$
\begin{aligned}
\sup _{b \in R^{+} \backslash U} & \inf _{t>0} E \exp \left\{t\left(2 b X-b^{2} X^{2}-x_{k}\right)\right\} \\
= & \left.\sup _{b>0} \inf _{t>0} E \exp \left\{t\left(2 b X-b^{2} X^{2}-x_{k}\right)\right\} \quad \text { [by (2.6) }\right] \\
\geq & \inf _{t>0} E \exp \left\{t\left(2 b_{k} X-b_{k}^{2} X^{2}-x_{k}\right)\right\} \\
\geq & \inf _{t>0}\left\{E \exp \left\{t\left(2 b_{k} X-b_{k}^{2} X^{2}-x_{k}\right)\right\} I\left(-x_{k} / b_{k} \leq X \leq 0\right)\right. \\
& \left.\quad+E \exp \left\{t\left(2 b_{k} X-b_{k}^{2} X^{2}-x_{k}\right)\right\} I\left(6 x_{k} /\left(p b_{k}\right) \leq X \leq 1 / b_{k}\right)\right\} \\
& \quad \inf _{t>0}\left\{e^{-4 t x_{k}} P\left(-x_{k} / b_{k} \leq X \leq 0\right)+e^{5 t x_{k} / p} P\left(6 x_{k} /\left(p b_{k}\right) \leq X \leq 1 / b_{k}\right)\right\} \\
= & P\left(-x_{k} / b_{k} \leq X \leq 0\right)+P\left(6 x_{k} /\left(p b_{k}\right) \leq X \leq 1 / b_{k}\right) \\
\rightarrow & 1
\end{aligned}
$$

by (2.9), where in the last equality, we used the fact that $e^{-4 t x_{k}} P\left(-x_{k} / b_{k} \leq X \leq\right.$ $0)+e^{5 t x_{k} / p} P\left(6 x_{k} /\left(p b_{k}\right) \leq X \leq 1 / b_{k}\right)$ is nondecreasing in $t>0$ for $k$ large enough. This proves (2.8) and hence the lemma.

Lemma 2.4. For $0<x<1$, we have

$$
\lim _{b \rightarrow \infty} \sup _{t>0} g(t, b ; x)=\infty \text { and } \lim _{b \rightarrow 0^{+}} \sup _{t>0} g(t, b ; x)=\infty .
$$

Proof. Let $k$ be a positive number. Then

$$
\begin{aligned}
\sup _{t>0} g(t, b ; x) & \geq g(k, b ; x) \\
& =k x-\ln E \exp \left\{-k\left(b^{2} X^{2}-2 b X\right)\right\} \\
& =k x-\ln E \exp \left\{-k(b X-1)^{2}+k\right\} .
\end{aligned}
$$

It follows from Lebesgue's dominated convergence theorem that

$$
\lim _{b \rightarrow \infty} E \exp \left\{-k(b X-1)^{2}+k\right\}=P(X=0) \text { and } \lim _{b \rightarrow 0^{+}} M(a)=1 .
$$

Combining (2.11)-(2.12) gives

$$
\liminf _{b \rightarrow \infty} \sup _{t>0} g(t, b ; x) \geq k x \text { and } \liminf _{b \rightarrow 0^{+}} \sup _{t>0} g(t, b ; x) \geq k x .
$$

This proves (2.10) by the arbitrariness of $k$.

We are now ready to prove Proposition 2.1.

Proof of Proposition 2.1. Lemmas 2.122.4 imply that $\inf _{b>0} \sup _{t \in R} g(t, b ; x)$ is attained at some finite points $b_{x} \in U$ and $t_{x}:=\tilde{t}\left(b_{x}, x\right)>0$ for all $x \in\left(0, \epsilon_{0}\right)$ when $\epsilon_{0}$ is sufficiently small. When $b \in U$, by Lemma 2.2(i), we have

$$
\frac{\partial g(\tilde{t}, b ; x)}{\partial t}=x-\frac{E Z e^{\tilde{t} Z}}{E e^{\tilde{t} Z}}=0, \quad \text { where } Z=2 b X-b^{2} X^{2} .
$$

Note that the assumption $\operatorname{Card}\left(C_{s}\right) \geq 3$ implies that $Z$ is nondegenerate; thus (A.2) is true. It then follows from the implicit function theorem that $\tilde{t}(b, x)$ is a differentiable function in some neighborhood $U^{*}(b)$ of $b$ (also a differentiable function in some neighborhood of $x$ ). We can also guarantee that $U^{*}(b) \subset U$. 
Hence $\sup _{t \in R} g(t, b ; x)$ is also a differentiable function in some neighborhood of $b_{x}$. So $b_{x}$ satisfies the equation $d g(\tilde{t}, b ; x) / d b=0$, i.e.,

$$
E\left(X-b X^{2}\right) \exp \left\{\tilde{t}\left(2 b X-b^{2} X^{2}\right)\right\}=0 .
$$

It follows from (2.13) and (2.14) that $b_{x}$ and $t_{x}$ are the solutions to the equations

$$
E Z e^{t Z}=x E e^{t Z}, \quad E X e^{t Z}=b E X^{2} e^{t Z} .
$$

Now we will show the uniqueness of $\left(t_{x}, b_{x}\right)$. Suppose $\left(t_{x}^{\prime}, b_{x}^{\prime}\right)$ is another point such that $g\left(t_{x}^{\prime}, b_{x}^{\prime} ; x\right)=\inf _{b>0} \sup _{t \in R} g(t, b ; x)$. Recall that

$$
g(t, b ; x)=-\ln E \exp \left\{t\left(2 b X-b^{2} X^{2}-x\right)\right\} .
$$

We must have

$$
\begin{aligned}
E \exp \left\{t_{x}\left(2 b_{x} X+b_{x}^{2} X^{2}-x\right)\right\} & =\sup _{b>0} E \exp \left\{t_{x}\left(2 b X+b^{2} X^{2}-x\right)\right\} \\
& \geq E \exp \left\{t_{x}\left(2 b_{x}^{\prime} X-b_{x}^{\prime 2} X^{2}-x\right)\right\} \\
& \geq \inf _{t>0} E \exp \left\{t\left(2 b_{x}^{\prime} X-b_{x}^{\prime 2} X^{2}-x\right)\right\} \\
& =E \exp \left\{t_{x}^{\prime}\left(2 b_{x}^{\prime} X-b_{x}^{\prime 2} X^{2}-x\right)\right\} .
\end{aligned}
$$

If $t_{x} \neq t_{x}^{\prime}$, then

$$
E \exp \left\{t_{x}\left(2 b_{x}^{\prime} X-b_{x}^{\prime 2} X^{2}-x\right)\right\}>\inf _{t>0} E \exp \left\{t\left(2 b_{x}^{\prime} X-b_{x}^{\prime 2} X^{2}-x\right)\right\}
$$

by the fact that $E \exp \left\{t\left(2 b X-b^{2} X^{2}-x\right)\right\}$ is a strictly convex function of $t$ for each fixed $b$ and $2 b X-b^{2} X^{2}-x$ is not identically equal to 0 . Combining (2.15) and (2.16) gives

$$
E \exp \left\{t_{x}\left(2 b_{x} X+b_{x}^{2} X^{2}-x\right)\right\}>E \exp \left\{t_{x}^{\prime}\left(2 b_{x}^{\prime} X-b_{x}^{\prime 2} X^{2}-x\right)\right\},
$$

which contradicts our assumption. Hence

$$
t_{x}=t_{x}^{\prime}
$$

Define

So we have

$$
f(b, s)=E \exp \left\{s\left(\frac{2}{b} X-X^{2}-\frac{x}{b^{2}}\right)\right\} .
$$

$$
f\left(b_{x}, s_{x}\right)=f\left(b_{x}^{\prime}, s_{x}^{\prime}\right)=\sup _{b>0} \inf _{s>0} f(b, s),
$$

where $s_{x}=t_{x} b_{x}^{2}, s_{x}^{\prime}=t_{x} b_{x}^{\prime 2}$. Noting that $f(b, s)$ is a strictly convex function of $s$ for each fixed $b$, similar to the proof of (2.17), we get $s_{x}=s_{x}^{\prime}$. Hence $b_{x}=b_{x}^{\prime}$. This completes the proof of uniqueness.

From now on, we assume $x \in\left(0, \epsilon_{0}\right)$ so that $\sup _{b>0} \inf _{t>0} E \exp \left\{t\left(2 b X-b^{2} X^{2}-x\right)\right\}$ is attained at the unique point $\left(t_{x}, b_{x}\right)$. The next proposition shows that, as a function of $x \in\left(0, \epsilon_{0}\right), E \exp \left\{t_{x}\left(2 b_{x} X-b_{x} X^{2}-x\right)\right\}$ is differentiable.

Proposition 2.2. Assume that $(H 1)$ and $(H 2)$ are satisfied and $\operatorname{Card}\left(C_{s}\right) \geq 3$. Then, $E \exp \left\{t_{x}\left(2 b_{x} X-b_{x}^{2} X^{2}-x\right)\right\}$ is a differentiable function of $x$ when $x \in\left(0, \epsilon_{0}\right)$.

The next two lemmas are needed to prove the proposition. We also assume that the condition of Proposition 2.2 is satisfied.

Lemma 2.5. $\forall x_{0} \in\left(0, \epsilon_{0}\right), b_{x}$ is a continuous function of $x$ in a neighborhood of $x_{0}$. 
Proof. Let $g(b, x)=\inf _{t>0} E \exp \left\{t\left(2 b X-b^{2} X^{2}-x\right)\right\}$. By (2.10), we have

$$
\lim _{b \rightarrow \infty} g(b, x)=0 \text { and } \lim _{b \rightarrow 0} g(b, x)=0 .
$$

Hence there exists some $\delta_{0}{ }^{\prime}>0$ such that if $x \in\left[x_{0}-\delta_{0}^{\prime}, x_{0}+\delta_{0}^{\prime}\right]$, then $b_{x} \in[L, U]$, where both $L$ and $U$ are positive constants depending only on $x_{0}$ and $\delta_{0}^{\prime}$. We can also choose $L$ and $U$ so that if $b<L$ or $b>U$, then

$$
\left|g\left(b, x_{0}\right)-g\left(b_{x_{0}}, x_{0}\right)\right| \geq \frac{1}{2} g\left(b_{x_{0}}, x_{0}\right) .
$$

Now we claim that $\exists \delta_{0}>0, \forall 0<\delta \leq \delta_{0}, \exists 0<\epsilon<g\left(b_{x_{0}}, x_{0}\right)$ such that

$$
\left\{b:\left|g\left(b, x_{0}\right)-g\left(b_{x_{0}}, x_{0}\right)\right| \leq \epsilon\right\} \subset\left(b_{x_{0}}-\delta, b_{x_{0}}+\delta\right) .
$$

Otherwise, $\exists$ a sufficiently small $\delta>0, \forall \epsilon>0$,

$$
\left\{b:\left|g\left(b, x_{0}\right)-g\left(b_{x_{0}}, x_{0}\right)\right| \leq \epsilon\right\} \cap\left(b_{x_{0}}-\delta, b_{x_{0}}+\delta\right)^{c} \neq \emptyset .
$$

So we will have a sequence $\left\{b_{m}\right\}_{m=1}^{\infty}$ such that $\left|b_{m}-b_{x_{0}}\right| \geq \delta$ for each $m \geq 1$ and $\lim _{m \rightarrow \infty} g\left(b_{m}, x_{0}\right)=g\left(b_{x_{0}}, x_{0}\right)$. (2.18) implies $\exists m_{0}>0$ such that if $m \geq m_{0}$, $b_{m} \in[L, U]$. Without loss of generality, we assume $b_{m} \rightarrow b_{x_{0}}^{\prime}$ as $m \rightarrow \infty$. The continuity of $g(b, x)$ gives

$$
g\left(b_{x_{0}}^{\prime}, x_{0}\right)=g\left(b_{x_{0}}, x_{0}\right) .
$$

Since $\left|b_{x_{0}}^{\prime}-b_{x_{0}}\right|>\delta$, (2.20) contradicts the fact that $b_{x_{0}}$ is unique. Therefore, we have (2.19). Note that

$$
\begin{aligned}
g(b, x) & =\inf _{t>0} E \exp \left\{t\left(2 b X-b^{2} X^{2}-x\right)\right\} \\
& =: \quad E \exp \left\{\tilde{t}(x, b)\left(2 b X-b^{2} X^{2}-x\right)\right\},
\end{aligned}
$$

where $\tilde{t}(x, b)$ is the unique solution to the following equation:

$$
E\left(2 b X-b^{2} X^{2}\right) \exp \left\{t\left(2 b X-b^{2} X^{2}\right)\right\}=x E \exp \left\{t\left(2 b X-b^{2} X^{2}\right)\right\},
$$

for each fixed $b$ and $x$. The implicit function theorem shows that $\tilde{t}(x, b)$ is a differentiable function of $x$ and $b$. Hence we can find some $0<\delta_{1} \leq \min \left(\delta_{0}, \delta_{0}^{\prime}\right)$ such that if $x \in\left[x_{0}-\delta_{1}, x_{0}+\delta_{1}\right]$,

$$
\left|g(b, x)-g\left(b, x_{0}\right)\right| \leq \frac{\epsilon}{2}
$$

uniformly in $b \in[L, U]$, and

$$
\left|g\left(b_{x}, x\right)-g\left(b_{x_{0}}, x_{0}\right)\right| \leq \frac{\epsilon}{2} .
$$

Combining (2.21) and (2.22) yields

$$
\left|g\left(b_{x}, x_{0}\right)-g\left(b_{x_{0}}, x_{0}\right)\right| \leq \epsilon .
$$

It follows from (2.19) and (2.23) that

$$
\left|b_{x}-b_{x_{0}}\right|<\delta,
$$

which shows the continuity of $b_{x}$ in some neighborhood of $x_{0}$.

Lemma 2.6. $t_{x}$ is an increasing and continuous function of $x$ in some neighborhood of $x_{0}$ for $x_{0} \in\left(0, \epsilon_{0}\right)$. 
Proof. Recall that $b_{x}$ and $t_{x}$ satisfy the following two equations:

$$
\begin{aligned}
& \frac{E Z e^{t Z}}{E e^{t Z}}=x, \\
& \frac{E\left(2 X-2 b X^{2}\right) e^{t Z}}{E e^{t Z}}=0,
\end{aligned}
$$

where $Z=2 b X-b^{2} X^{2}$. Write

$$
\begin{aligned}
& a_{1}(b, t)=\frac{E Z^{2} e^{t Z}}{E e^{t Z}}-\left(\frac{E Z e^{t Z}}{E e^{t Z}}\right)^{2} \\
& a_{2}(b, t)=\frac{E Z\left(2 X-2 b X^{2}\right) e^{t Z}}{E e^{t Z}}, \\
& a_{3}(b, t)=\frac{E\left(2 X-2 b X^{2}\right)^{2} e^{t Z}}{E e^{t Z}} t-2 \frac{E X^{2} e^{t Z}}{E e^{t Z}} .
\end{aligned}
$$

Since $b_{x}$ is a supremum point of $g(b, x)$, we have

$$
a_{3}\left(b_{x}, t_{x}\right) \leq 0 .
$$

Also

$$
a_{1}\left(b_{x}, t_{x}\right)>0
$$

because $2 b_{x} X-b_{x}^{2} X^{2}$ is nondegenerate. If $a_{3}\left(b_{x_{0}}, t_{x_{0}}\right)<0$ or $a_{2}\left(b_{x_{0}}, t_{x_{0}}\right) \neq 0$, then

$$
\left.\frac{d t_{x}}{d x}\right|_{x=x_{0}}=\frac{a_{3}\left(b_{x_{0}}, t_{x_{0}}\right)}{a_{1}\left(b_{x_{0}}, t_{x_{0}}\right) a_{3}\left(b_{x_{0}}, t_{x_{0}}\right)-a_{2}^{2}\left(b_{x_{0}}, t_{x_{0}}\right)} \geq 0
$$

by the implicit function theorem. So in this case, $t_{x}$ is increasing and continuous at $x=x_{0}$.

If $a_{3}\left(b_{x_{0}}, t_{x_{0}}\right)=0$ and $a_{2}\left(b_{x_{0}}, t_{x_{0}}\right)=0$, then there exists some neighborhood of $x_{0}$ such that $a_{3}\left(b_{x}, t_{x}\right)<0$ or $a_{2}\left(b_{x}, t_{x}\right) \neq 0$ when $x \in U \backslash\left\{x_{0}\right\}$. Otherwise, we find some sequence $\left\{x_{n}\right\}_{n=1}^{\infty}$ which goes to $x_{0}$ as $n \rightarrow \infty$ such that

$$
a_{3}\left(b_{x_{n}}, t_{x_{n}}\right)=0 \text { and } a_{2}\left(b_{x_{n}}, t_{x_{n}}\right)=0 .
$$

Since $a_{3}(b, t)$ and $a_{2}(b, t)$ are infinitely differentiable functions of $b$ and $t$,

$$
a_{3}(b, t) \equiv 0 \text { and } a_{2}(b, t) \equiv 0
$$

when $(b, t)^{T}$ belongs to some neighborhood $U_{1}$ of $\left(b_{x_{0}}, t_{x_{0}}\right)^{T}$. Noting that $\left(b_{x_{0}}, t_{x_{0}}\right)^{T}$ satisfies (2.25), we can conclude that

$$
E\left(2 X-2 b X^{2}\right) e^{t Z} \equiv 0
$$

when $(b, t)^{T} \in U_{1}$. Hence

$$
E \exp \left\{t_{x_{0}}\left(2 b X-b^{2} X^{2}\right)\right\} \equiv E \exp \left\{t_{x_{0}}\left(2 b_{x_{0}} X-b_{x_{0}}^{2} X^{2}\right)\right\}
$$

when $b$ is in some neighborhood of $b_{x_{0}}$. But (2.27) contradicts the uniqueness of $b_{x_{0}}$. So

$$
\frac{d t_{x}}{d x} \geq 0 \text { if } x \in U \backslash\left\{x_{0}\right\},
$$

which, combined with Lemma 2.5 and the fact that $\tilde{t}(x, b)$ determined by (2.24) is a continuous function of $x$ and $b$, implies $t_{x}$ is increasing and continuous at $x=x_{0}$. 
Proof of Proposition 2.2. Let

$e(x)=\int_{x_{0}}^{x}\left(-t_{y}\right) E \exp \left\{t_{y}\left(2 b_{y} X-b_{y}^{2} X^{2}-y\right)\right\} d y+E \exp \left\{t_{x_{0}}\left(2 b_{x_{0}} X-b_{x_{0}}^{2} X^{2}-x_{0}\right)\right\}$.

Then

$$
\frac{d e(x)}{d x}=\left(-t_{x}\right) E \exp \left\{t_{x}\left(2 b_{x} X-b_{x}^{2} X^{2}-x\right)\right\} .
$$

We also know that $E \exp \left\{t_{x}\left(2 b_{x} X-b_{x}^{2} X^{2}-x\right)\right\}$ is differentiable a.e. in $\left(0, \epsilon_{0}\right)$, and

$$
\frac{d}{d x} E \exp \left\{t_{x}\left(2 b_{x} X-b_{x}^{2} X^{2}-x\right)\right\}=-t_{x} E \exp \left\{t_{x}\left(2 b_{x} X-b_{x}^{2} X^{2}-x\right)\right\} \text { a.e. }
$$

So

$$
e(x)=E \exp \left\{t_{x}\left(2 b_{x} X-b_{x}^{2} X^{2}-x\right)\right\} \text { a.e. }
$$

Since both $e(x)$ and $E \exp \left\{t_{x}\left(2 b_{x} X-b_{x}^{2} X^{2}-x\right)\right\}$ are continuous functions of $x$ by Lemmas 2.5-2.6.

$$
e(x)=E \exp \left\{t_{x}\left(2 b_{x} X-b_{x}^{2} X^{2}-x\right)\right\},
$$

which gives the final assertion.

\section{Convergence of $b_{x}$ And $t_{x}$}

Proposition 3.1. Assume that (H1) and (H2) are satisfied and that $\operatorname{Card}\left(C_{s}\right) \geq 3$. Then

$$
\lim _{x \rightarrow 0^{+}} b_{x}=0 .
$$

Proof. We will show that for any sequence $\left\{x_{n}\right\}_{n=1}^{\infty}$ decreasing to 0 , it is impossible that

$$
\begin{aligned}
& \lim _{n \rightarrow \infty} b_{x_{n}}=\infty, \\
& \lim _{n \rightarrow \infty} b_{x_{n}}=b_{0},
\end{aligned}
$$

where $b_{0}$ is some positive constant. Now we suppose (3.2). Then

$$
\begin{aligned}
\inf _{t>0} E \exp \left\{t\left(2 b_{x_{n}} X-b_{x_{n}}^{2} X^{2}-x_{n}\right)\right\} & =\inf _{t>0} E \exp \left\{-t\left(b_{x_{n}} X-1\right)^{2}+t\left(1-x_{n}\right)\right\} \\
& \leq E \exp \left\{-c\left(b_{x_{n}} X-1\right)^{2}+t_{0}\left(1-x_{n}\right)\right\} \\
& \rightarrow P(X=0),
\end{aligned}
$$

as $n \rightarrow \infty$, where $c$ is any positive constant. But the above result contradicts Lemma 8.1 of Shao [14.

It remains to exclude (3.3). So we assume (3.3). Equation (1.7) gives

$$
\frac{E\left(2 X-b_{x_{n}} X^{2}\right) \exp \left\{s_{n}\left(2 X-b_{x_{n}} X^{2}\right)\right\}}{E \exp \left\{s_{n}\left(2 X-b_{x_{n}} X^{2}\right)\right\}}=\frac{x_{n}}{b_{n}},
$$

where $s_{n}=t_{x_{n}} b_{x_{n}}$. Lemma 2.6 combined with (3.3), shows $\lim _{n \rightarrow \infty} s_{n}=s_{0}$, where $s_{0}=t_{0} b_{0}$. Letting $n \rightarrow \infty$ in (3.4) yields

$$
2 E X \exp \left\{s_{0}\left(2 X-b_{0} X^{2}\right)\right\}=b_{0} E X^{2} \exp \left\{s_{0}\left(2 X-b_{0} X^{2}\right)\right\} \text {, }
$$

which contradicts another equation (1.8)

$$
E\left(X-b_{x_{n}} X^{2}\right) \exp \left\{s_{n}\left(2 X-b_{x_{n}} X^{2}\right\}=0 .\right.
$$


Proposition 3.2. Assume that (H1), (H2) and (1.5) are satisfied and that $\operatorname{Card}\left(C_{s}\right)$ $\geq 3$. Then

$$
\lim _{x \rightarrow 0^{+}} t_{x}=t_{0}>0
$$

Proof. Convergence of $t_{x}$ follows from Lemma 2.6. So we need to show $t_{0}>0$. Otherwise, suppose $t_{0}=0$. Write $Z_{x}=2 b_{x} X-b_{x}^{2} X^{2}$ and $D_{x}=t_{x}^{-1 / 3}$. Then

$$
\begin{aligned}
& \left|E b_{x} X e^{t_{x} Z_{x}} I\left(\left|b_{x} X\right| \leq D_{x}\right)-E b_{x} X I\left(\left|b_{x} X\right| \leq D_{x}\right)\right| \\
\leq & \epsilon_{x} E\left(b_{x} X\right)^{2} e^{t_{x} Z_{x}} I\left(\left|b_{x} X\right| \leq D_{x}\right),
\end{aligned}
$$

where $\epsilon_{x} \rightarrow 0$ as $x \rightarrow 0^{+}$. Since $X \in \mathcal{F}_{\theta}$ for some $\theta<\infty$, we have for $x$ sufficiently close to 0 ,

$$
\begin{aligned}
& E b_{x} X e^{t_{x} Z_{x}} I\left(\left|b_{x} X\right| \leq D_{x}\right) \\
& \quad \leq E b_{x} X I\left(\left|b_{x} X\right| \leq D_{x}\right)+\epsilon_{x} E\left(b_{x} X\right)^{2} e^{t_{x} Z_{x}} I\left(\left|b_{x} X\right| \leq D_{x}\right) \\
& \quad \leq \frac{\theta+1}{D_{x}} E\left(b_{x} X\right)^{2} I\left(\left|b_{x} X\right| \leq D_{x}\right)+\epsilon_{x} E\left(b_{x} X\right)^{2} e^{t_{x} Z_{x}} I\left(\left|b_{x} X\right| \leq D_{x}\right) \\
& \quad \leq\left(\frac{\theta+\epsilon^{\prime}}{D_{x}} e^{t_{x}\left(2 D_{x}+D_{x}^{2}\right)}+\epsilon_{x}\right) E\left(b_{x} X\right)^{2} e^{t_{x} Z_{x}} I\left(\left|b_{x} X\right| \leq D_{x}\right) \\
& \quad<(1 / 4) E\left(b_{x} X\right)^{2} e^{t_{x} Z_{x}} I\left(\left|b_{x} X\right| \leq D_{x}\right) .
\end{aligned}
$$

On the other hand,

$$
\begin{aligned}
& 2 E b_{x} X e^{t_{x} Z_{x}} I\left(\left|b_{x} X\right|>D_{x}\right) \\
< & E\left(b_{x} X\right)^{2} e^{t_{x} Z_{x}} I\left(\left|b_{x} X\right|>D_{x}\right)
\end{aligned}
$$

for $x$ sufficiently small.

Combining (3.6) and (3.7), we have

$$
E Z_{x} e^{t_{x} Z_{x}}<0
$$

for $x$ sufficiently close to 0 , but this contradicts the saddlepoint equation

$$
E\left(Z_{x}-x\right) e^{t_{x} Z_{x}}=0 .
$$

We shall also need the following lemma.

Lemma 3.1. Assume that (H1), (H2) and (1.5) are satisfied and that $\operatorname{Card}\left(C_{s}\right) \geq$ 3. Write $Z_{x}=2 b_{x} X-b_{x}^{2} X^{2}$. Then

$$
\begin{aligned}
& E e^{t_{x} Z_{x}}=1+o(x), \\
& E\left(b_{x} X\right)^{2} e^{t_{x} Z_{x}}=x(1+o(x)),
\end{aligned}
$$

as $x \rightarrow 0^{+}$.

Proof. From the proof of Proposition 2.2, we see that

$$
\frac{d}{d x} E \exp \left\{t_{x}\left(Z_{x}-x\right)\right\}=-t_{x} E \exp \left\{t_{x}\left(Z_{x}-x\right)\right\} .
$$


Hence

$$
E \exp \left\{t_{x}\left(Z_{x}-x\right)\right\}=\exp \left(-\int_{0}^{x} t_{y} d y\right)
$$

which combined with Proposition 3.1 gives

$$
\begin{aligned}
E e^{t_{x} Z_{x}} & =E \exp \left\{t_{x}\left(Z_{x}-x\right)\right\} e^{x t_{x}} \\
& =\exp \left(\int_{0}^{x}\left(t_{x}-t_{y}\right) d y\right) \\
& =\exp (o(x))=1+o(x) .
\end{aligned}
$$

By Equations (1.7) and (1.8),

$$
\begin{aligned}
E\left(b_{x} X\right)^{2} e^{t_{x} Z_{x}} & =x E e^{t_{x} Z_{x}} \\
& =x(1+o(x)) .
\end{aligned}
$$

\section{Proof of MAIN THEOREMS}

Write

$$
\begin{gathered}
K(x)=x^{-2} E X^{2} I(|X| \leq x) \text { for } x>0 \\
d(t)=\inf \left\{s \geq a_{0}+1: K(s) \leq 1 / t\right\}, \text { where } a_{0}=\inf \{x \geq 1: K(x)>0\} .
\end{gathered}
$$

4.1. Proof of Theorem 1.1. If $\operatorname{Card}\left(C_{s}\right) \leq 2$, then $X$ takes only two possible values. In this case, (1.4) is a direct consequence of Theorem 3.1 of Shao [14. If $\operatorname{Card}\left(C_{s}\right) \geq 3$, then (1.4) follows from (1.6). In fact, by Lemma 3.1 (with $t_{n}=t_{x_{n}^{2} / n}$ and $\left.b_{n}=b_{x_{n}^{2} / n}\right)$

$$
\begin{aligned}
n \lambda\left(x_{n}^{2} / n\right) & =t_{n} x_{n}^{2}-n \ln E \exp \left(t_{n}\left(2 b_{n} X-b_{n}^{2} X^{2}\right)\right) \\
& =t_{n} x_{n}^{2}-n \ln \left(1+o\left(x_{n}^{2} / n\right)\right)=t_{n} x_{n}^{2}+o\left(x_{n}^{2}\right)=t_{0} x_{n}^{2}+o\left(x_{n}^{2}\right) .
\end{aligned}
$$

Thus we only need to prove (1.6).

Upper bound proof. Let $\delta$ be an arbitrarily small but fixed constant, $B$ an arbitrarily large but fixed constant. Both $\delta$ and $B$ will be specified later. Then

$$
\begin{aligned}
P\left(\frac{S_{n}}{V_{n}} \geq x_{n}\right) & \leq P\left(\frac{S_{n}}{V_{n}} \geq x_{n}, 0 \leq V_{n} \leq \frac{1}{\delta} a_{n}\right)+P\left(\frac{S_{n}}{V_{n}} \geq x_{n}, V_{n}>\frac{1}{\delta} a_{n}\right) \\
& :=T_{1}+T_{2},
\end{aligned}
$$

where $a_{n}$ is some number depending on $n$. We deal with $T_{2}$ first. Let $z_{n}$ be some positive number which will be defined later. By the Cauchy-Schwarz inequality, $\left|\sum_{i=1}^{n} X_{i} I\left(\left|X_{i}\right| \geq z_{n}\right)\right| \leq V_{n}\left(\sum_{i=1}^{n} I\left(\left|X_{i}\right| \leq z_{n}\right)\right)^{1 / 2}$ and hence

$$
\begin{aligned}
T_{2} & \leq P\left(\sum_{i=1}^{n} X_{i} I\left(\left|X_{i}\right| \leq z_{n}\right) \geq \frac{x_{n}}{2 \delta} a_{n}\right)+P\left(\sum_{i=1}^{n} X_{i} I\left(\left|X_{i}\right| \geq z_{n}\right) \geq \frac{x_{n}}{2} V_{n}\right) \\
& \leq P\left(\sum_{i=1}^{n} X_{i} I\left(\left|X_{i}\right| \geq z_{n}\right) \geq \frac{x_{n}}{2 \delta} a_{n}\right)+P\left(\sum_{i=1}^{n} I\left(\left|X_{i}\right| \leq z_{n}\right) \geq \frac{x_{n}^{2}}{4}\right) \\
& :=T_{21}+T_{22} .
\end{aligned}
$$


For $T_{21}$, Bernstein's inequality gives

$$
T_{21} \leq 2 \exp \left(-\frac{n\left(x_{n} a_{n} /(2 n \delta)-E X I\left(|X| \leq z_{n}\right)\right)^{2}}{2 \operatorname{Var} X I\left(|X| \leq z_{n}\right)+\frac{4}{3} z_{n}\left(x_{n} a_{n} /(2 n \delta)-E X I\left(|X| \leq z_{n}\right)\right)}\right) .
$$

Applying the Chernoff large deviation to the binomial random variable $B(n, p)$, it follows that for all $a>0$,

$$
P(B(n, p)>a n) \leq\left(\frac{e p}{a}\right)^{a n} .
$$

Therefore

$$
T_{22} \leq\left(\frac{12 P\left(|X| \geq z_{n}\right)}{x_{n}^{2} / n}\right)^{x_{n}^{2} / 4}
$$

In the following, we will analyze the exponent in (4.2) and the ratio in (4.3), respectively. Let

$$
z_{n}:=\inf \left\{x \geq a_{0}+1:\left(2 x^{2}\right)^{-1} E X^{2} I(|X| \leq x) \leq \frac{\delta^{2}}{B^{2}} \frac{x_{n}^{2}}{n}\right\} .
$$

Then $z_{n}=d\left(1 /\left(2 \frac{\delta^{2}}{B^{2}} \frac{x_{n}^{2}}{n}\right)\right) \rightarrow \infty$ as $n \rightarrow \infty$.

Define $a_{n}=x_{n} z_{n} / B$. Hence for $n$ sufficiently large,

$$
\begin{aligned}
\frac{x_{n} a_{n}}{2 n \delta}-E X I\left(|X| \leq z_{n}\right) & \geq \frac{1}{B \delta}\left(\frac{1}{2}-\frac{4 \theta \delta^{3}}{B}\right) \frac{x_{n}^{2}}{n} z_{n}, \\
\frac{n\left(x_{n} a_{n} /(2 n \delta)-E X I\left(|X| \leq z_{n}\right)\right)^{2}}{2 \operatorname{Var} X I\left(|X| \leq z_{n}\right)} & \geq \frac{B}{4 \delta^{3}}\left(\frac{1}{2}-\frac{4 \theta \delta^{3}}{B}\right)^{2} x_{n}^{2} \\
\frac{n\left(x_{n} a_{n} /(2 n \delta)-E X I\left(|X| \leq z_{n}\right)\right)}{4 z_{n} / 3} & \geq \frac{3}{4 B \delta}\left(\frac{1}{2}-\frac{4 \theta \delta^{3}}{B}\right) x_{n}^{2} \\
\frac{12 P\left(|X| \geq z_{n}\right)}{x_{n}^{2} / n} & \leq 30 \theta \frac{\delta^{2}}{B^{2}} .
\end{aligned}
$$

Hence we can select suitable $B$ and $\delta$ so that $B \delta$ is very small, which ensures that $T_{21}$ and $T_{22}$ are negligible relative to $\exp \left(-t_{n} x_{n}^{2}\right)$. So we have

$$
\frac{\ln T_{2}}{t_{n} x_{n}^{2}} \rightarrow-\infty
$$

Now let's deal with $T_{1}$. Let $u_{n}=z_{n} /\left(\delta B x_{n}^{2}\right)$. Noting that $a_{n} /\left(\delta x_{n}^{2}\right)=u_{n} x_{n}$ and that $0 \leq c \leq v \leq h$ implies

$$
v \geq\left(v^{2}+c h\right) /(c+h),
$$


we have for $s>0$,

$$
\begin{aligned}
T_{1} & =P\left(\frac{S_{n}}{V_{n}} \geq x_{n}, 0 \leq V_{n} \leq \frac{1}{\delta} a_{n}\right) \\
& \leq \sum_{i=0}^{\left[x_{n}^{2}\right]} P\left(\frac{S_{n}}{x_{n}} \geq V_{n}, \frac{i a_{n}}{\delta x_{n}^{2}} \leq V_{n} \leq \frac{(i+1) a_{n}}{\delta x_{n}^{2}}\right) \\
& \leq \sum_{i=0}^{\left[x_{n}^{2}\right]} P\left((2 i+1) u_{n} S_{n} \geq V_{n}^{2}+i(i+1) u_{n}^{2} x_{n}^{2}\right) \\
& \leq \sum_{i=0}^{\left[x_{n}^{2}\right]}\left(E \exp \left\{s\left((2 i+1) u_{n} X-X^{2}-i(i+1) u_{n}^{2} x_{n}^{2} / n\right)\right\}^{n}\right) \\
& =\sum_{i=0}^{\left[x_{n}^{2}\right]}\left(E \exp \left\{s\left((2 i+1) u_{n} X-X^{2}-(i+1 / 2)^{2} u_{n}^{2} x_{n}^{2} / n\right)+(s / 4) u_{n}^{2} x_{n}^{2} / n\right\}^{n}\right) .
\end{aligned}
$$

Since

$$
\begin{aligned}
E & \exp \left\{s\left((2 i+1) u_{n} X-X^{2}-(i+1 / 2)^{2} u_{n}^{2} x_{n}^{2} / n\right)\right\} \\
& =E \exp \left\{\frac{s}{(i+1 / 2)^{2} u_{n}^{2}}\left(\frac{2}{(i+1 / 2) u_{n}} X-\frac{1}{(i+1 / 2)^{2} u_{n}^{2}} X^{2}-x_{n}^{2} / n\right)\right\} \\
& \leq \sup _{b \geq 0} E \exp \left\{\frac{s}{b^{2}}\left(2 b X-b^{2} X^{2}-x_{n}^{2} / n\right)\right\},
\end{aligned}
$$

we have

$$
T_{1} \leq\left(1+\left[x_{n}^{2}\right]\right)\left(E \exp \left\{t_{n}\left[2 b_{n} X-b_{n}^{2} X^{2}-\frac{x_{n}^{2}}{n}\right]+t_{n} b_{n}^{2} u_{n}^{2} x_{n}^{2} / n\right\}\right)^{n},
$$

where $t_{n}$ and $b_{n}$ are chosen such that

$$
E \exp \left\{t_{n}\left(2 b_{n} X-b_{n}^{2} X^{2}-x_{n}^{2} / n\right)\right\}:=\sup _{b \geq 0} \inf _{t \geq 0} E \exp \left\{t\left(2 b X-b^{2} X^{2}-x_{n}^{2} / n\right)\right\},
$$

or $t_{n}:=t_{x_{n}^{2} / n}, b_{n}:=b_{x_{n}^{2} / n}$. Recall that $t_{n}$ and $b_{n}$ satisfy

$$
\frac{E\left(b_{n} X\right)^{2} e^{t_{n}\left(2 b_{n} X-b_{n}^{2} X^{2}\right)}}{E e^{t_{n}\left(2 b_{n} X-b_{n}^{2} X^{2}\right)}}=\frac{x_{n}^{2}}{n} .
$$

Hence for $\epsilon$ close enough to 0 , we have

$$
\begin{aligned}
& E\left(b_{n} X\right)^{2} I\left(\left|b_{n} X\right| \leq \epsilon\right) \\
\leq & E\left(b_{n} X\right)^{2} e^{t_{n}\left(2 b_{n} X-b_{n}^{2} X^{2}\right)} I\left(\left|b_{n} X\right| \leq \epsilon\right) e^{t_{n}\left(2 \epsilon+\epsilon^{2}\right)} \\
\leq & 2 x_{n}^{2} / n,
\end{aligned}
$$

where we used Lemma 3.1 in the last inequality. Hence

$$
d\left(\frac{\epsilon^{2}}{2} \frac{n}{x_{n}^{2}}\right) \leq \frac{\epsilon^{2}}{b_{n}} .
$$


Combining Lemma 3.1 of Griffin and Kuelbs [10] and (4.7), we have for $n$ sufficiently large,

$$
\begin{aligned}
d\left(\frac{B^{2} n}{2 \delta^{2} x_{n}^{2}}\right) / \frac{\epsilon^{2}}{b_{n}} & \leq d\left(\frac{B^{2} n}{2 \delta^{2} x_{n}^{2}}\right) / d\left(\frac{\epsilon^{2}}{2} \frac{n}{x_{n}^{2}}\right) \\
& \leq\left(\frac{B^{2}(1+\theta)}{\epsilon^{2} \delta^{2}}\right)^{\frac{1+\theta}{2}},
\end{aligned}
$$

i.e.

$$
b_{n} z_{n} \leq \epsilon^{2}\left(\frac{B^{2}(1+\theta)}{\epsilon \delta^{2}}\right)^{\frac{1+\theta}{2}} .
$$

Combining (4.1), (4.5), (4.6), (4.8), Proposition 2.2 and Proposition 3.2 gives that, $\forall \epsilon_{0}>0$,

$$
\frac{\ln T_{1}}{x_{n}^{2}} \leq-t_{0}+\epsilon_{0}
$$

for $n$ sufficiently large. Therefore

$$
\limsup _{n \rightarrow \infty} \frac{\ln P\left(\frac{S_{n}}{V_{n}} \geq x_{n}\right)}{x_{n}^{2}} \leq-t_{0}
$$

Lower bound proof. By the basic equality $|x y|=\inf _{b \geq 0}(2 b)^{-1}\left(b^{2} x^{2}+y^{2}\right)$, we have

$$
\begin{aligned}
P\left(\frac{S_{n}}{V_{n}} \geq x_{n}\right) & =P\left(S_{n} \geq \inf _{b \geq 0} \frac{1}{2 b}\left(b^{2} V_{n}^{2}+x_{n}^{2}\right)\right) \\
& =P\left(\sup _{b \geq 0} \sum_{i=1}^{n}\left(2 b X_{i}-b^{2} X_{i}^{2}-\frac{x_{n}^{2}}{n}\right) \geq 0\right) \\
& \geq P\left(\sum_{i=1}^{n}\left(2 b_{n} X_{i}-b_{n}^{2} X_{i}^{2}-\frac{x_{n}^{2}}{n}\right) \geq 0\right) .
\end{aligned}
$$

Tilting methods give

$$
\begin{aligned}
& P\left(\sum_{i=1}^{n}\left(2 b_{n} X_{i}-b_{n}^{2} X_{i}^{2}-\frac{x_{n}^{2}}{n}\right) \geq 0\right) \\
= & \left(E \exp \left\{t_{n}\left(Z_{n}-\frac{x_{n}^{2}}{n}\right)\right\}\right)^{n} \int_{0}^{\infty} e^{-t_{n} \sigma\left(t_{n}\right) y \sqrt{n}} d F_{n}(y),
\end{aligned}
$$

where $Z_{n}=2 b_{n} X-b_{n}^{2} X^{2}$,

$$
\sigma^{2}\left(t_{n}\right)=\frac{E Z_{n}^{2} e^{t_{n} Z_{n}}}{E e^{t_{n} Z_{n}}}-\left(\frac{E Z_{n} e^{t_{n} Z_{n}}}{E e^{t_{n} Z_{n}}}\right)^{2}
$$

$F_{n}(y)$ is the distribution function of the random variable $\sum_{i=1}^{n}\left(\eta_{i}-E \eta_{i}\right) / \sqrt{n \sigma^{2}\left(t_{n}\right)}$, and $\eta_{1}, \eta_{2}, \ldots, \eta_{n}$ are i.i.d. r.v.'s with distribution function

$$
V(x)=\frac{1}{E e^{t_{n} Z_{n}}} \int_{-\infty}^{x} e^{t_{n} y} d P\left(Z_{n} \leq y\right) .
$$


By Lemma 4.2 in Section 4.3, we have

$$
\begin{aligned}
& \int_{0}^{\infty} \exp \left\{-t_{n} \sigma\left(t_{n}\right) y \sqrt{n}\right\} d F_{n}(y) \\
\geq & \int_{0}^{2} \exp \left\{-t_{n} \sigma\left(t_{n}\right) y \sqrt{n}\right\} d F_{n}(y) \\
\geq & \exp \left\{-2 t_{n} \sigma\left(t_{n}\right) \sqrt{n}\right\} P\left(0 \leq \sum_{i=1}^{n}\left(\eta_{i}-E \eta_{i}\right) / \sqrt{n \sigma^{2}\left(t_{n}\right)} \leq 2\right) \\
\geq & 2^{-1}(\Phi(2)-\Phi(0)) \exp \left\{-2 t_{n} \sigma\left(t_{n}\right) \sqrt{n}\right\}
\end{aligned}
$$

for $n$ sufficiently large. Hence

$$
\begin{aligned}
& P\left(\frac{S_{n}}{V_{n}} \geq x_{n}\right) \\
\geq & 2^{-1}(\Phi(2)-\Phi(0)) \exp \left\{-2 t_{n} \sigma\left(t_{n}\right) \sqrt{n}\right\}\left(E \exp \left\{t_{n}\left(Z_{n}-\frac{x_{n}^{2}}{n}\right)\right\}\right)^{n} .
\end{aligned}
$$

Now we will prove

$$
t_{n} \sigma\left(t_{n}\right) \sqrt{n}=o\left(x_{n}^{2}\right)
$$

as $n \rightarrow \infty$.

Let $\epsilon$ be some small positive constant. Then since $X \in \mathcal{F}_{\theta}$,

$$
\begin{aligned}
\epsilon^{2} P\left(\left|b_{n} X\right| \geq \epsilon\right) & \leq 2 \theta E\left(b_{n} X\right)^{2} I\left(\left|b_{n} X\right| \leq \epsilon\right) \\
& \leq 2 \theta E\left(b_{n} X\right)^{2} e^{t_{n}\left(Z_{n}+4 \epsilon\right)} I\left(\left|b_{n} X\right| \leq \epsilon\right) \\
& \leq c \frac{x_{n}^{2}}{n}
\end{aligned}
$$

where (4.12) follows from Lemma 3.1 and $c$ is some positive constant. Therefore (4.11) and (4.12) imply

$$
\begin{aligned}
& t_{n}^{2} E Z_{n}^{2} e^{t_{n} Z_{n}} \\
= & t_{n}^{2} E Z_{n}^{2} e^{t_{n} Z_{n}} I\left(\left|b_{n} X\right| \leq \epsilon\right)+t_{n}^{2} E Z_{n}^{2} e^{t_{n} Z_{n}} I\left(\left|b_{n} X\right|>\epsilon\right) \\
\leq & (2+\epsilon)^{2} t_{n}^{2} E\left(b_{n} X\right)^{2} e^{t_{n} Z_{n}} I\left(\left|b_{n} X\right| \leq \epsilon\right)+e P\left(\left|b_{n} X\right|>\epsilon\right) \\
\leq & c_{1} \frac{x_{n}^{2}}{n}
\end{aligned}
$$

for some constant $c_{1}>0$. So

$$
\begin{aligned}
t_{n} \sigma\left(t_{n}\right) \sqrt{n} & \leq\left(\frac{n t_{n}^{2} E Z_{n}^{2} e^{t_{n} Z_{n}}}{E e^{t_{n} Z_{n}}}\right)^{\frac{1}{2}} \\
& \leq \sqrt{c_{1} x_{n}} / \sqrt{E e^{t_{n} Z_{n}}} \\
& =o\left(x_{n}^{2}\right),
\end{aligned}
$$

where the last equality is from Lemma 3.1. So we completed the proof of (4.10). Noting that Lemma 3.1 gives, $\forall \epsilon_{1}>0$,

$$
E \exp \left\{t_{n}\left(Z_{n}-\frac{x_{n}^{2}}{n}\right)\right\} \geq \exp \left\{\left(-t_{0}-\epsilon_{1}\right) \frac{x_{n}^{2}}{n}\right\}
$$


for $n$ sufficiently large, we have

$$
\liminf _{n \rightarrow \infty} \frac{\ln P\left(S_{n} / V_{n} \geq x_{n}\right)}{x_{n}^{2}} \geq-t_{0}-\epsilon_{1}
$$

by (4.9) and (4.10). The arbitrariness of $\epsilon_{1}$ completes the lower bound proof.

4.2. Proof of Theorem 1.2. When $\operatorname{Card}\left(C_{s}\right) \leq 2$, the result is due to Theorem 5.1 of Shao [14. When $\operatorname{Card}\left(C_{s}\right) \geq 3$, following the proof of Theorem 1.1](similar to Proposition 5.1 in Shao [14], see Appendix), one can prove that under the condition of Theorem 1.1] for any $0<\varepsilon<1 / 2$, there exists $\tau>1$ such that

$$
P\left(\max _{n \leq k \leq \tau n} \frac{S_{k}}{V_{k}} \geq x_{n}\right) \leq \exp \left(-(1-\varepsilon) t_{0} x_{n}^{2}\right) .
$$

The rest of the proof is similar to that of Theorem 5.1 in Shao [14 by the subsequence method. The details are omitted here.

\subsection{Auxiliary lemmas.}

Lemma 4.1. Write $Z_{n}=2 b_{n} X-b_{n}^{2} X^{2}$. Then

$$
\liminf _{n \rightarrow \infty} \frac{E Z_{n}^{2} e^{t_{n} Z_{n}}}{x_{n}^{2} / n}>c_{0}>0
$$

where $c_{0}$ is some constant.

Proof. First, we show that for arbitrary $\epsilon_{2}>0$,

$$
\liminf _{n \rightarrow \infty} \frac{E b_{n}^{2} X^{2} I\left(\left|b_{n} X\right| \leq \epsilon_{2}\right)}{x_{n}^{2} / n}=c_{2}>0 .
$$

Otherwise, there exists $\epsilon_{3}>0$ such that

$$
\liminf _{n \rightarrow \infty} \frac{E b_{n}^{2} X^{2} I\left(\left|b_{n} X\right| \leq \epsilon_{3}\right)}{x_{n}^{2} / n}=0 .
$$

Without loss of generality, we can assume

$$
\lim _{n \rightarrow \infty} \frac{E b_{n}^{2} X^{2} I\left(\left|b_{n} X\right| \leq \epsilon_{3}\right)}{x_{n}^{2} / n}=0 .
$$

Since $X \in \mathcal{F}_{\theta}$, we have

$$
\lim _{n \rightarrow \infty} \frac{P\left(\left|b_{n} X\right|>\epsilon_{3}\right)}{x_{n}^{2} / n}=0
$$

Hence

$$
\begin{aligned}
& \lim _{n \rightarrow \infty} \frac{E\left(b_{x} X\right)^{2} e^{t_{x} Z_{x}} I\left(\left|b_{n} X\right|>\epsilon_{3}\right)}{x_{n}^{2} / n} \\
\leq & \lim _{n \rightarrow \infty} \frac{c_{2 n} P\left(\left|b_{n} X\right|>\epsilon_{3}\right)}{x_{n}^{2} / n} \\
= & 0,
\end{aligned}
$$

where $c_{2 n}=\sup _{|y|>\epsilon_{3}} y^{2} \exp \left\{t_{n}\left(2 y-y^{2}\right)\right\}$, whose limit is positive by Proposition 3.2 as $n \rightarrow \infty$. Combining (4.16) with (3.9), we get for $n$ sufficiently large,

$$
\begin{aligned}
E b_{n}^{2} X^{2} I\left(\left|b_{n} X\right| \leq \epsilon_{3}\right) & \geq e^{-t_{n}\left(2 \epsilon_{3}+\epsilon_{3}^{2}\right)} E\left(b_{x} X\right)^{2} e^{t_{x} Z_{x}} I\left(\left|b_{n} X\right| \leq \epsilon_{3}\right) \\
& \geq \frac{1}{2} \frac{x_{n}^{2}}{n}
\end{aligned}
$$


which contradicts our assumption. Therefore 4.15) holds. Now

$$
\begin{aligned}
& \liminf _{n \rightarrow \infty} \frac{E Z_{n}^{2} e^{t_{n} Z_{n}}}{x_{n}^{2} / n} \\
\geq & \liminf _{n \rightarrow \infty} \frac{E Z_{n}^{2} e^{t_{n} Z_{n}}}{x_{n}^{2} / n} I\left(\left|b_{n} X\right| \leq \epsilon_{2}\right) \\
\geq & \liminf _{n \rightarrow \infty}\left(2-\epsilon_{2}\right)^{2} \exp \left\{-t_{n}\left(2 \epsilon_{3}+\epsilon_{3}^{2}\right)\right\} \frac{E b_{n}^{2} X^{2} I\left(\left|b_{n} X\right| \leq \epsilon_{2}\right)}{x_{n}^{2} / n} \\
= & \left(2-\epsilon_{2}\right)^{2} \exp \left\{-t_{0}\left(2 \epsilon_{2}+\epsilon_{2}^{2}\right)\right\} c_{2} \\
> & 0,
\end{aligned}
$$

which is just (4.14).

\section{Lemma 4.2.}

$$
\sum_{i=1}^{n}\left(\eta_{i}-E \eta_{i}\right) / \sqrt{n \sigma^{2}\left(t_{n}\right)} \rightarrow N(0,1)
$$

in distribution as $n \rightarrow \infty$.

Proof. It suffices to show the Lindeberg condition

$$
\frac{E Z_{n}^{2} e^{t_{n} Z_{n}} I\left(\left|Z_{n}\right| \geq \delta \sqrt{n} \sigma\left(t_{n}\right)\right)}{\sigma^{2}\left(t_{n}\right)} \rightarrow 0
$$

holds as $n \rightarrow \infty$ for arbitrary $\delta>0$.

Lemma 4.1 yields $\sqrt{n} \sigma\left(t_{n}\right) \rightarrow \infty$ as $n \rightarrow \infty$. So

$$
\begin{aligned}
& \sup _{y}\left(2 b_{n} y-b_{n}^{2} y^{2}\right)^{2} \exp \left\{t_{n}\left(2 b_{n} y-b_{n}^{2} y^{2}\right)\right\} I\left(\left|2 b_{n} y-b_{n}^{2} y^{2}\right| \geq \delta \sqrt{n} \sigma\left(t_{n}\right)\right) \\
= & \delta^{2} n \sigma^{2}\left(t_{n}\right) \exp \left\{-t_{n} \delta \sqrt{n} \sigma\left(t_{n}\right)\right\} .
\end{aligned}
$$

Write $\delta_{n}=t_{n} \delta \sqrt{n} \sigma\left(t_{n}\right)$. Hence for $n$ sufficiently large,

$$
\begin{aligned}
& \frac{E Z_{n}^{2} e^{t_{n} Z_{n}} I\left(\left|Z_{n}\right| \geq \delta \sqrt{n} \sigma\left(t_{n}\right)\right)}{\sigma^{2}\left(t_{n}\right)} \\
\leq & \delta^{2} n e^{-\delta_{n}} P\left(\left|Z_{n}\right| \geq \delta \sqrt{n} \sigma\left(t_{n}\right)\right) \\
\leq & \delta^{2} n e^{-\delta_{n}} P\left(\left|b_{n} X\right| \geq 1\right) \\
\leq & \delta^{2} n e^{-\delta_{n}} c_{3} \frac{x_{n}^{2}}{n} \\
\leq & c_{3} \delta^{2} x_{n}^{2} \exp \left\{-t_{n} \delta \sqrt{c_{0}} \sqrt{n} x_{n} / 2\right\} \\
\rightarrow & 0,
\end{aligned}
$$

where (4.17) is due to the Feller class assumption and the saddlepoint equations, and the last inequality follows from Lemma 4.1. The proof of asymptotic normality is complete. 


\section{Appendix}

Proof of Lemma 2.1. It suffices to show that $g(t, b ; x)$ is strictly increasing in $t$, either

(I) for $t \in(-\infty, 0] ; \quad$ or $\quad(I I)$ for $t \in\left(0, \epsilon_{1}\right]$ for some $\epsilon_{1}>0$.

We prove $(I)$ first. Let $Z=2 b X-b^{2} X^{2}$. For arbitrary $t$ and $t_{1}$ such that $t_{1}<t \leq 0$, we need to show that $g\left(t_{1}, b ; x\right)<g(t, b ; x)$. If $E e^{t_{1} Z}=\infty$, then $g\left(t_{1}, b ; x\right)=t_{1} x-\ln E e^{t_{1} Z}=-\infty$, in which case $(I)$ follows straightaway. Now assume that $E e^{t_{1} Z}<\infty$ below, which implies that moments of $X$ of all orders exist. Thus, $g(t, b ; x)$ is differentiable in $t$ for $t \in\left(t_{1}, \infty\right)$. Taking derivatives gives

$$
\frac{\partial g(t, b ; x)}{\partial t}=x-\frac{E Z e^{t Z}}{E e^{t Z}}
$$

Observe that

$$
\left.\frac{\partial g(t, b ; x)}{\partial t}\right|_{t=0}=x+b^{2} E X^{2}>0
$$

and

$$
\frac{\partial^{2} g(t, b ; x)}{\partial t^{2}}=-\left(\frac{E Z^{2} e^{t Z}}{E e^{t Z}}-\left(\frac{E Z e^{t Z}}{E e^{t Z}}\right)^{2}\right)<0,
$$

since $Z=2 b X+b^{2} X^{2}$ is nondegenerate by the assumption that $\operatorname{Card}\left(C_{s}\right) \geq 3$ and $E X=0$ or $E X^{2}=\infty$. Thus, $\frac{\partial g(t, b ; x)}{\partial t}>0$ when $t \in\left(t_{1}, 0\right]$. So $g(t, b ; x)$ is strictly increasing in $\left(t_{1}, 0\right]$. Since $t_{1}$ is arbitrary, we have hence proved $(I)$.

We prove $(I I)$ next. If there exists some $t_{2}<0$ such that $E e^{t_{2} Z}<\infty$, then (II) follows from the fact that $\frac{\partial g(0, b ; x)}{\partial t}=x+b^{2} E X^{2}>0$. It remains to prove $(I I)$ under the condition that

$$
E e^{t_{3} Z}=\infty, \quad \text { for all } t_{3}<0 .
$$

To show this, we choose an arbitrary $t>0$. Then from (A.1), we have

$$
\frac{\partial g(t, b ; x)}{\partial t}=x-\frac{\int_{-\infty}^{\infty}\left(2 b y-b^{2} y^{2}\right) e^{-t(b y-1)^{2}} d F(y)}{\int_{-\infty}^{\infty} e^{-t(b y-1)^{2}} d F(y)} .
$$

The monotone convergence theorem implies

$$
\begin{aligned}
\lim _{t \rightarrow 0^{+}} \int_{-\infty}^{\infty} e^{-t(b y-1)^{2}} d F(y) & =1, \\
\lim _{t \rightarrow 0^{+}} \int_{-\infty}^{\infty} y^{2} e^{-t(b y-1)^{2}} d F(y) & =E X^{2} \quad \text { (it could be } \infty \text { ), }
\end{aligned}
$$

where $t \rightarrow 0^{+}$means that $t \rightarrow 0$ from the right side of 0 .

If $E X^{2}=\infty$, then

$$
\begin{aligned}
& \lim _{t \rightarrow 0^{+}} \int_{-\infty}^{\infty}\left(2 b y-b^{2} y^{2}\right) e^{-t(b y-1)^{2}} d F(y) \\
\leq & \lim _{t \rightarrow 0^{+}} \int_{-\infty}^{\infty}\left(4+\frac{b^{2} y^{2}}{4}-b^{2} y^{2}\right) e^{-t(b y-1)^{2}} d F(y) \\
= & 4-\frac{3}{4} b^{2} E X^{2} \\
= & -\infty
\end{aligned}
$$


If $E X^{2}<\infty$, then noting $\left|y e^{-t(b y-1)^{2}}\right| \leq|y|$ for $t>0$, we can use Lebesgue's dominated convergence theorem to get

$$
\lim _{t \rightarrow 0^{+}} \int_{-\infty}^{\infty}(2 b y) e^{-t(b y-1)^{2}} d F(y)=2 b E X=0 .
$$

Combining (A.3)-(A.7) gives

$$
\lim _{t \rightarrow 0^{+}} \frac{\partial g(t, b ; x)}{\partial t}>0
$$

Noting that $g(t, b ; x)$ is right continuous at $t=0$, we conclude $(I I)$.

Proof of Equation (4.13). Let $\eta=\varepsilon / 20$. Clearly,

$$
\begin{aligned}
& P\left(\max _{n \leq k \leq \tau n} \frac{S_{k}}{V_{k}} \geq x_{n}\right) \\
& \quad \leq P\left(\frac{S_{n}}{V_{n}} \geq(1-5 \eta) x_{n}\right)+P\left(\max _{n<k \leq \tau n} \frac{S_{k}-S_{n}}{V_{k}} \geq 5 \eta x_{n}\right) .
\end{aligned}
$$

By Theorem 1.1, we have

$$
P\left(\frac{S_{n}}{V_{n}} \geq(1-5 \eta) x_{n}\right) \leq \exp \left(-(1-\varepsilon / 2) t_{0} x_{n}^{2}\right)
$$

provided that $n$ is sufficiently large.

Below we estimate the second term on the right hand side of (A.8). Similar to (4.3) and (4.4) in Shao [14, let

$$
\begin{gathered}
l(x)=E X^{2} I\{|X| \leq x\}, \quad b=\inf \{x \geq 1: l(x)>0\}, \\
z_{n}=\inf \left\{s: s \geq b+1, \frac{l(s)}{s^{2}} \leq \frac{16\left(t_{0}+t_{0}^{2}\right) x_{n}^{2}}{\eta^{2} n}\right\} .
\end{gathered}
$$

By an elementary argument and the assumption that $x_{n}^{2}=o(n)$, it is plain to see that

(A.11) $z_{n} \rightarrow \infty$ and $n l\left(z_{n}\right)=16\left(t_{0}+t_{0}^{2}\right) x_{n}^{2} z_{n}^{2} / \eta^{2}$ for every $n$ sufficiently large.

Since $X$ is in the centered Feller class, there exists $C>0$ such that for all $x>b+1$,

$$
P(|X|>x) \leq C x^{-2} l(x)
$$

and

$$
|E X I(|X| \leq x)| \leq C x^{-1} l(x) .
$$


Write

$$
\begin{aligned}
P\left(\max _{n<k \leq \tau n} \frac{S_{k}-S_{n}}{V_{k}} \geq 5 \eta x_{n}\right) \\
\leq \quad P\left(\max _{n<k \leq \tau n} \frac{\sum_{i=n+1}^{k} X_{i} I\left\{\left|X_{i}\right| \leq z_{n}\right\}}{V_{k}} \geq 4 \eta x_{n}\right) \\
\quad+P\left(\max _{n<k \leq \tau n} \frac{\sum_{i=n+1}^{k}\left|X_{i}\right| I\left\{\left|X_{i}\right| \geq z_{n}\right\}}{V_{k}} \geq \eta x_{n}\right) \\
\leq \quad P\left(\max _{n<k \leq \tau n} \sum_{i=n+1}^{k} X_{i} I\left\{\left|X_{i}\right| \leq z_{n}\right\} \geq 2 \eta x_{n} \sqrt{n l\left(z_{n}\right)}\right) \\
\quad+P\left(V_{n} \leq \sqrt{n l\left(z_{n}\right)} / 2\right)+P\left(\sum_{i=n+1}^{[\tau n]} I\left\{\left|X_{i}\right| \geq z_{n}\right\} \geq\left(\eta x_{n}\right)^{2}\right) .
\end{aligned}
$$

Note that for independent nonnegative random variables $\left\{\xi_{i}\right\}$ with finite second moments, we have (see, e.g., Lemma 2.1 of Einmahl and Mason [5])

$$
P\left(\sum_{i=1}^{n} \xi_{i} \leq x\right) \leq \exp \left(-\frac{(\mu-x)^{2}}{2 \sigma^{2}}\right)
$$

for $0<x<\mu$, where $\mu=\sum_{i=1}^{n} E \xi_{i}$ and $\sigma^{2}=\sum_{i=1}^{n} E \xi_{i}^{2}$. Using (A.14), we have

$$
\begin{aligned}
P & \left(V_{n} \leq \sqrt{n l\left(z_{n}\right)} / 2\right) \\
& \leq P\left(\sum_{i=1}^{n} X_{i}^{2} I\left(\left|X_{i}\right| \leq z_{n}\right) \leq n l\left(z_{n}\right) / 4\right) \\
& \leq \exp \left(-\frac{\left(0.75 n l\left(z_{n}\right)\right)^{2}}{2 n E X^{4} I\left(|X| \leq z_{n}\right)}\right) \\
& \leq \exp \left(-\frac{0.75^{2} n l\left(z_{n}\right)}{2 z_{n}^{2}}\right) \\
& \leq \exp \left(-4.5 t_{0} x_{n}^{2}\right) \quad \text { by (A.11). }
\end{aligned}
$$

It is known that for a binomial random variable $B(n, p)$ with parameters $n$ and $p$ (see, e.g., [Dudley [4], p.16])

$$
P(B(n, p)>a n) \leq\left(\frac{e p}{a}\right)^{a n} \text { for } a>0 .
$$


Hence we have

$$
\begin{aligned}
& P\left(\sum_{i=n+1}^{[\tau n]} I\left\{\left|X_{i}\right| \geq z_{n}\right\} \geq\left(\eta x_{n}\right)^{2}\right) \\
& \quad \leq\left(\frac{3(\tau-1) n P\left(|X| \geq z_{n}\right)}{\left(\eta x_{n}\right)^{2}}\right)^{\left(\eta x_{n}\right)^{2}} \\
& \quad \leq\left(\frac{3(\tau-1) n C z_{n}^{-2} l\left(z_{n}\right)}{\left(\eta x_{n}\right)^{2}}\right)^{\left(\eta x_{n}\right)^{2}} \\
& \quad \leq\left(3(\tau-1) 16\left(t_{0}+t_{0}^{2}\right) / \eta^{2}\right)^{\left(\eta x_{n}\right)^{2}} \\
& \quad \leq \exp \left(-2 t_{0} x_{n}^{2}\right),
\end{aligned}
$$

as long as $\tau$ is very close to one.

By A.13 and A.11), if $\tau-1>0$ is chosen to be sufficiently small,

$$
\sum_{i=n+1}^{[\tau n]}\left|E X_{i} I\left\{\left|X_{i}\right| \leq z_{n}\right\}\right| \leq C(\tau-1) n l\left(z_{n}\right) / z_{n} \leq \frac{1}{2} \eta x_{n} \sqrt{n l\left(z_{n}\right)}
$$

and

$$
\sum_{i=n+1}^{[\tau n]} \operatorname{Var} X_{i} I\left\{\left|X_{i}\right| \leq z_{n}\right\} \leq(\tau-1) n l\left(z_{n}\right) \leq \frac{1}{8} \eta n x_{n}^{2} l\left(z_{n}\right) .
$$

Therefore, by the Ottaviani maximum inequality and the Bernstein exponential inequality,

$$
\begin{aligned}
& P\left(\max _{n<k \leq \tau n} \sum_{i=n+1}^{k} X_{i} I\left\{\left|X_{i}\right| \leq z_{n}\right\} \geq 2 \eta x_{n} \sqrt{n l\left(z_{n}\right)}\right) \\
& \quad \leq 2 P\left(\sum_{i=n+1}^{[\tau n]} X_{i} I\left\{\left|X_{i}\right| \leq z_{n}\right\}-E X_{i} I\left\{\left|X_{i}\right| \leq z_{n}\right\} \geq \eta x_{n} \sqrt{n l\left(z_{n}\right)}\right) \\
& \leq 2 \exp \left(-\frac{\eta^{2} x_{n}^{2} n l\left(z_{n}\right)}{2\left((\tau-1) n l\left(z_{n}\right)+\eta x_{n} \sqrt{n l\left(z_{n}\right)} z_{n}\right)}\right) \\
& \leq 2 \exp \left(-\frac{\eta^{2} x_{n}^{2} n l\left(z_{n}\right)}{4(\tau-1) n l\left(z_{n}\right)}\right)+2 \exp \left(-\frac{\eta x_{n} \sqrt{n l\left(z_{n}\right)}}{4 z_{n}}\right) \\
& \leq 2 \exp \left(-\eta^{2} x_{n}^{2} /(4(\tau-1))\right)+\exp \left(-4 t_{0} x_{n}^{2}\right) \\
& \leq 4 \exp \left(-4 t_{0} x_{n}^{2}\right)
\end{aligned}
$$

provided $(\tau-1)>0$ is sufficiently small. Putting together the above inequalities yields

$$
P\left(\max _{n<k \leq \tau n} \frac{S_{k}-S_{n}}{V_{k}} \geq 5 \eta x_{n}\right) \leq 6 \exp \left(-2 t_{0} x_{n}^{2}\right) .
$$

This proves (4.13), by (A.9), (A.8) and (A.15).

\section{ACKNOWLEDGEMENT}

We thank an anonymous referee for insightful comments. 


\section{REFERENCES}

1. V. Bentkus and F. Götze. The Berry-Esseen bound for Student's statistic. Ann. Probab. 24 (1996), 491-503. MR:1387647 (97f:62021)

2. M. Csörgö, B. Szyszkowicz and Q. Wang. Darling-Erdős theorem for self-normalized sums. Ann. Probab. 31 (2003), 676-692. MR1964945(2004a:60051)

3. M. Csörgő, B. Szyszkowicz and Q. Wang. Donsker's theorem for self-normalized partial sums processes. Ann. Probab. 31 (2003), 1228-1240. MR.1988470 (2004h:60031)

4. R.M. Dudley. Uniform Central Limit Theorems. Cambridge Studies in Advanced Mathematics, Vol. 63. Cambridge University Press, Cambridge, 1999. MR1720712 (2000k:60050)

5. U. Einmahl and D.M. Mason. Some universal results on the behavior of increments of partial sums. Ann. Probab. 24 (1996), 1388-1407. MR.1411499 (97m:60034)

6. E. Giné, F. Götze and D. M. Mason. When is the Student $t$-statistic asymptotically standard normal? Ann. Probab. 25 (1997), 1514-1531. MR1457629 (98j:60033)

7. E. Giné and D. M. Mason. On the LIL for self-normalized sums of IID random variables. $J$. Theoret. Probab. 11 (1998), 351-370. MR.1622575(99e:60082)

8. F. Götze and G. P. Chistyakov. Limit distributions of studentized means. Ann. Probab. 32 (2004), 28-77. MR2040775 (2005f:60055)

9. P. S. Griffin. Tightness of the Student $t$-statistic. Electron. Comm. Prob. 7 (2002), 171-180. MR.1937903 (2003i:60037)

10. P. S. Griffin and J. Kuelbs. Self-normalized laws of the iterated logarithm. Ann. Probab. 17 (1989), 1571-1601. MR1048947 (91k:60036)

11. B.-Y. Jing, Q. M. Shao and Q. Wang. Self-normalized Cramér-type large deviations for independent random variables. Ann. Probab. 31 (2003), 2167-2215. MR2016616 (2004k:60069)

12. B.-Y. Jing, Q. M. Shao and W. Zhou. Saddlepoint approximation for Student's $t$-statistic with no moment conditions. Ann. Statist. 32 (2004), 2679-2711. MR2153999(2006a:62030)

13. B. F. Logan, C. L. Mallows, S. O. Rice and L. A. Shepp. Limit distributions of self-normalized sums. Ann. Probab. 1 (1973), 788-809. MR0362449(50:14890)

14. Q. M. Shao. Self-normalized large deviations. Ann. Probab. 25 (1997), 285-328. MR1428510 (98b:60056)

15. Q. M. Shao. Recent developments in self-normalized limit theorems. In Asymptotic Methods in Probability and Statistics (editor B. Szyszkowicz), pp. 467 - 480. Elsevier Science, 1998. MR.1661499 (99j:60063)

16. Q. M. Shao. Recent progress on self-normalized limit theorems. Probability, Finance and Insurance. Edited by T.L. Lai, H. Yang, and S.P. Yung, World Scientific, 2004. MR2189198 (2006i:60056)

Department of Mathematics, Hong Kong University of Science and Technology, HONG KONG

E-mail address: majing@ust.hk

Department of Mathematics, Hong Kong University of Science and Technology, Hong Kong - and - Department of Mathematics, University of Oregon, Eugene, Oregon 97403

E-mail address: maqmshao@ust.hk

Department of Statistics and Applied Probability, National University of SingaPORE, SingaPORE 117546

E-mail address: stazw@nus.edu.sg 